\title{
Corrigendum to "A Comparison between Piezoelectric Devices and Conventional Rotary Instruments in Bone Harvesting in Patients with Lip and Palate Cleft: A Retrospective Study with Clinical, Radiographical, and Histological Evaluation"
}

\author{
R. Rullo, ${ }^{1}$ A. Piccirillo, ${ }^{2}$ F. Femiano, ${ }^{1}$ L. Nastri, ${ }^{3}$ and V. M. Festa $\mathbb{D}^{2}$ \\ ${ }^{1}$ Associate Professor, Multidisciplinary Department of Medical-Surgical and Dental Specialties, \\ University of Campania Luigi Vanvitelli, Naples, Italy \\ ${ }^{2}$ Resident of Oral and Maxillofacial Surgery, Multidisciplinary Department of Medical-Surgical and Dental Specialties, \\ University of Campania Luigi Vanvitelli, Naples, Italy \\ ${ }^{3}$ Aggregate Professor, Multidisciplinary Department of Medical-Surgical and Dental Specialties, \\ University of Campania Luigi Vanvitelli, Naples, Italy \\ Correspondence should be addressed to V. M. Festa; vincenzomariafesta@gmail.com
}

Received 4 October 2018; Accepted 8 October 2018; Published 22 October 2018

Copyright (C) 2018 R. Rullo et al. This is an open access article distributed under the Creative Commons Attribution License, which permits unrestricted use, distribution, and reproduction in any medium, provided the original work is properly cited.

In the article titled "A Comparison between Piezoelectric Devices and Conventional Rotary Instruments in Bone Harvesting in Patients with Lip and Palate Cleft: A Retrospective Study with Clinical, Radiographical, and Histological Evaluation" [1], an acknowledgment should be added as follows:

The authors would like to acknowledge the Valere Project of the University of Campania Luigi Vanvitelli for the support to publish this article.

\section{References}

[1] R. Rullo, A. Piccirillo, F. Femiano, L. Nastri, and V. M. Festa, "A Comparison between Piezoelectric Devices and Conventional Rotary Instruments in Bone Harvesting in Patients with Lip and Palate Cleft: A Retrospective Study with Clinical, Radiographical, and Histological Evaluation," BioMed Research International, vol. 2018, Article ID 2059464, 8 pages, 2018. 


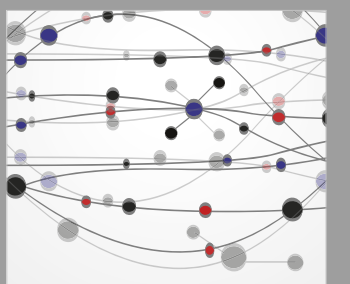

The Scientific World Journal
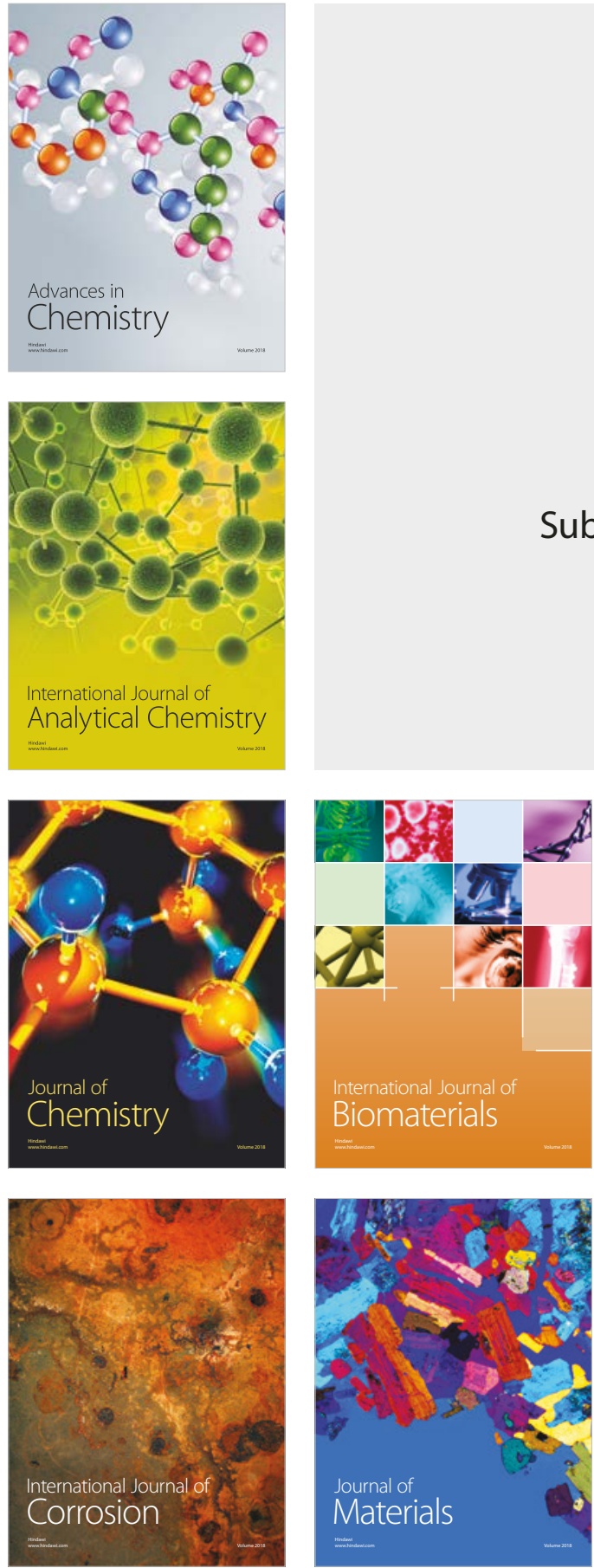

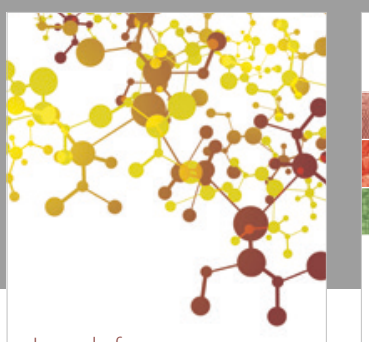

Journal of

Applied Chemistry
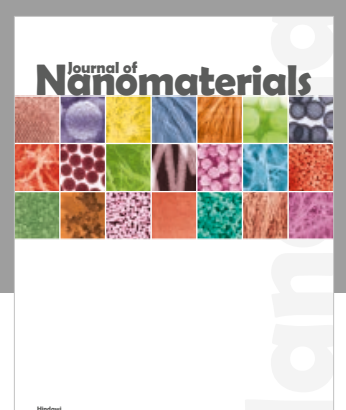

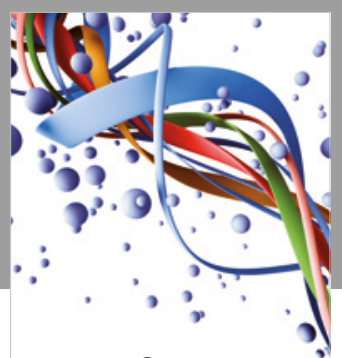

Scientifica

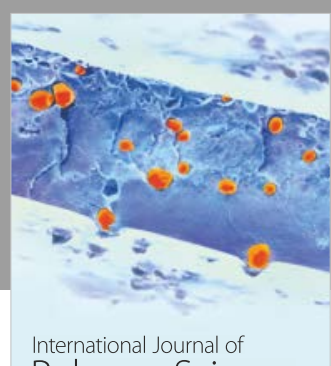

Polymer Science

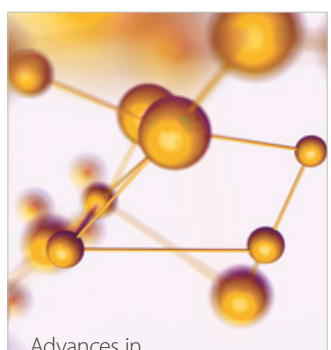

Physical Chemistry
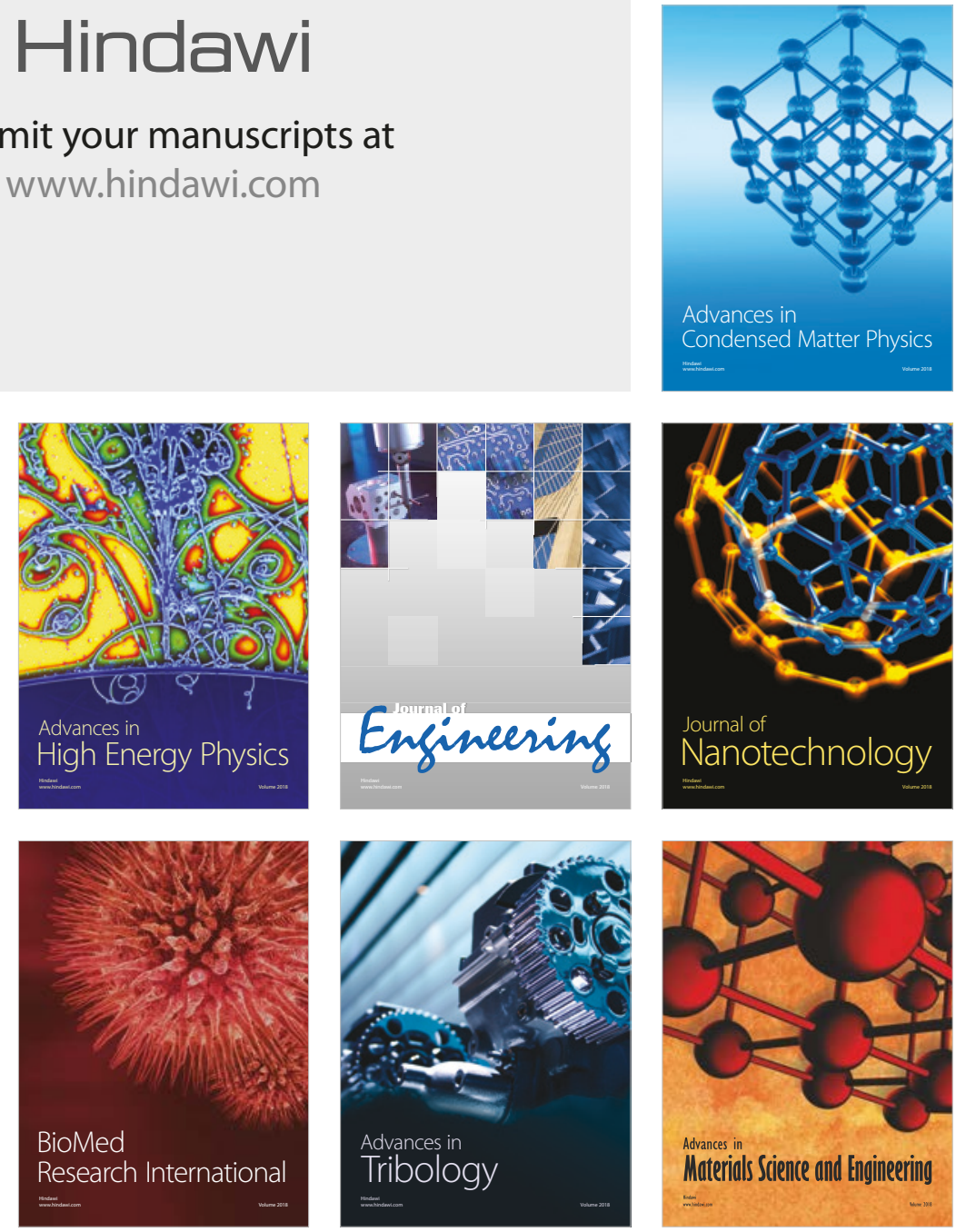